\title{
ANALYSIS OF DOSE RATES AROUND THE SLOVENIAN SILO-TYPE LILW REPOSITORY USING ADVANTG
}

\author{
Domen Kotnik ${ }^{1,2}$, Bor Kos ${ }^{1,2}$, Dušan Čalič ${ }^{1}$, and Luka Snoj ${ }^{1,2}$ \\ ${ }^{1}$ Reactor Physics Department, Jožef Stefan Institute, Jamova cesta 39, 1000 Ljubljana, Slovenia \\ ${ }^{2}$ Faculty of mathematics and physics, University of Ljubljana, Jadranska ulica 19, 1000 \\ Ljubljana, Slovenia \\ domen.kotnik@ijs.si,bor.kos@ijs.si,dusan.calic@ijs.si, luka.snoj@ijs.si
}

\begin{abstract}
The ADVANTG code was used to analyze dose rates from the proposed Slovenian silo-type low and intermediate level waste (LILW) repository. Detailed calculations of dose rates are challenging as gamma-sources are located in thick concrete containers and the effect of backscattering in the air has to be considered. Compared to analog MCNP simulations maximum relative speed-up of up to 9500 was achieved by using ADVANTG. We have analyzed the influence of different repository configurations on the final annual $\gamma$-dose rates. For different measurement positions, which were located from $10 \mathrm{~m}$ to $150 \mathrm{~m}$ from the outer edge of the repository, $\gamma$-dose rates were calculated. The results show that for all configurations of the open repository where the waste from different sources is deposited, except using the waste from Krško NPP, the calculated annual $\gamma$-dose rates for all measurement positions were lower than the prescribed limit values for professionals of $20 \mathrm{mSv} / \mathrm{year}$. To ensure that the $\gamma$ dose rates are lower than the prescribed limit values even for the case with the waste from Krško NPP an additional analysis was made where the concrete casks, in which the waste is stored, were modelled in more detail and the quantity of additional grout on top of the waste was varied.
\end{abstract}

KEYWORDS: ADVANTG, variance reduction, LILW repository

\section{INTRODUCTION}

A low and intermediate level waste (LILW) repository is planned to be constructed in Slovenia [1]. It will contain existing and future LILW produced in Slovenia originating from medicine, industry, research reactor and waste due to decommissioning of the Krško NPP [2] and the research reactor JSI TRIGA Mark II [3]. This unique silo-type repository will be regularly filled over a long period ( $\sim 40$ years). The radioactive waste will be stored in standardized concrete containers (called $\mathrm{N} 2 \mathrm{~b}$ type) and placed into the repository by layers. Each layer will contain 99 containers, which at full capacity of ten layers represents 990 containers. The repository is assumed to be filled with the waste in approximately 2065 . After that, it will be fully closed and covered with an additional layer of concrete and clay at the top. Before that, the repository will be open and therefore a thorough analysis of dose rates around the repository should be performed.

The use of Monte Carlo (MC) particle transport methods is desired as the major contribution to dose rates around the repository is due to "skyshine" effect. Moreover, the additional approach of using variance 
reduction methods is essential due to high level of $\gamma$ ray flux attenuation from the source inside, which is located inside of the concrete containers, to the detectors on the surface around the repository. To study dose rates and the effect of various physical parameters on them the state-of-the-art Monte Carlo code (MCNP [4]) was used for gamma-ray transport. ADVANTG [5-6] was used to generate variance reduction parameters to speed up MCNP calculations in terms of required CPU time.

In our previous analyses, the ADVANTG code has been successfully validated and evaluated on two ICSBEP benchmark experiments; the "Labyrinth" shielding benchmark (ALARM-CF-AIR-LAB-001 [7], i.e. the neutron flux in a concrete labyrinth [8]) and the "Skyshine" benchmark (ALARM-REAC-AIRSKY-001 [9], i.e. neutron/gamma-dose fields from an open working research reactor [10]). The skyshine benchmark experiment serves as a validated basis for all ADVANTG accelerated calculations performed in this paper as it is a similar problem to the LILW repository case.

In the first part of the paper computational methods, models and description of the radiation source are presented. In the second part the calculations of $\gamma$ dose rates around the repository, evaluation of the contribution of individual layers of LILW, and studying the effect of different parameters, i.e. $\gamma$ ray energy, repository filling fraction and waste type, to the total dose rate are presented. All the calculations were performed using the hybrid methodology, i.e. ADVANTG accelerated MCNP simulations.

\section{COMPUTATIONAL METHODS AND MODELS}

\subsection{MCNP and ADVANTG}

The Monte Carlo N-Particle Transport Code (MCNP) is a general-purpose, generalized-geometry, continuous-energy and time-dependent code that can be used for coupled neutron/gamma-transport and other particle transport simulations developed by the Los Alamos National Laboratory. Gamma-ray transport calculations were performed using MCNP5 ver. 1.6 code and ENDF/B-VII.1 [11] nuclear data library.

ADVANTG, an AutomateD VAriaNce reducTion Generator [5-6], is a code developed by the Oak Ridge National Laboratory, which automatically generates variance reduction parameters, defined in the MCNP input file, for neutron and/or gamma-transport problems. ADVANTG generates energy- and spacedependent mesh-based weight windows (WW) parameters, and a biased source distribution based on an approximate deterministic solution of the adjoint and forward transport equation. So-called hybrid method combines positive aspects of MC and deterministic methods (MCNP + ADVNATG). The basic concept is to increase the rate of tally convergence by using variance reduction parameters and therefore to accelerate analog MC simulations in terms of required CPU time.

\subsection{The model of the LILW repository}

The simplified model of the repository used in the calculations is shown in Fig. 1. It represents a fully loaded open concrete silo type storage of a cylindrical structure with a height of $49 \mathrm{~m}$ and the internal diameter of $27.5 \mathrm{~m}$, where each layer contains 99 containers. The thickness of the reinforced concrete wall and the bottom were $2.2 \mathrm{~m}$ and $3.5 \mathrm{~m}$, respectively. Measurement positions (MP), 12 spheres with a radius of $2 \mathrm{~m}$ at a height of $2 \mathrm{~m}$ from the ground, were placed at ground level. Our goal was to simulate only backscattered $\gamma$-rays from the air (skyshine effect) and not direct $\gamma$-rays from the source, therefore, the MP are located at distances from $10 \mathrm{~m}$ to $150 \mathrm{~m}$ from the outer edge of the repository (the expected size of the LILW site, i.e. the distance from the source to the perimeter fence, is $160 \mathrm{~m}$ ). The model used in the calculations includes $300 \mathrm{~m}$ of air above the surface, the ground with the thickness of $62.5 \mathrm{~m}(10 \mathrm{~m}$ thick layer below the bottom of concrete repository) and an axial length of $250 \mathrm{~m}$ from the boundaries to the outer edge of the repository (last MP is at a distance of $150 \mathrm{~m}$ from the outer edge of the repository). Across 
the entire geometry of the problem, both air and ground layers were modelled homogeneously with a constant density. The chosen optimal height of modelled air, $300 \mathrm{~m}$, was based on a detailed analysis. It turned out, that $\gamma$ dose rates deviated less than $1 \%$ for all MPs compared to the case with $800 \mathrm{~m}$ of air.

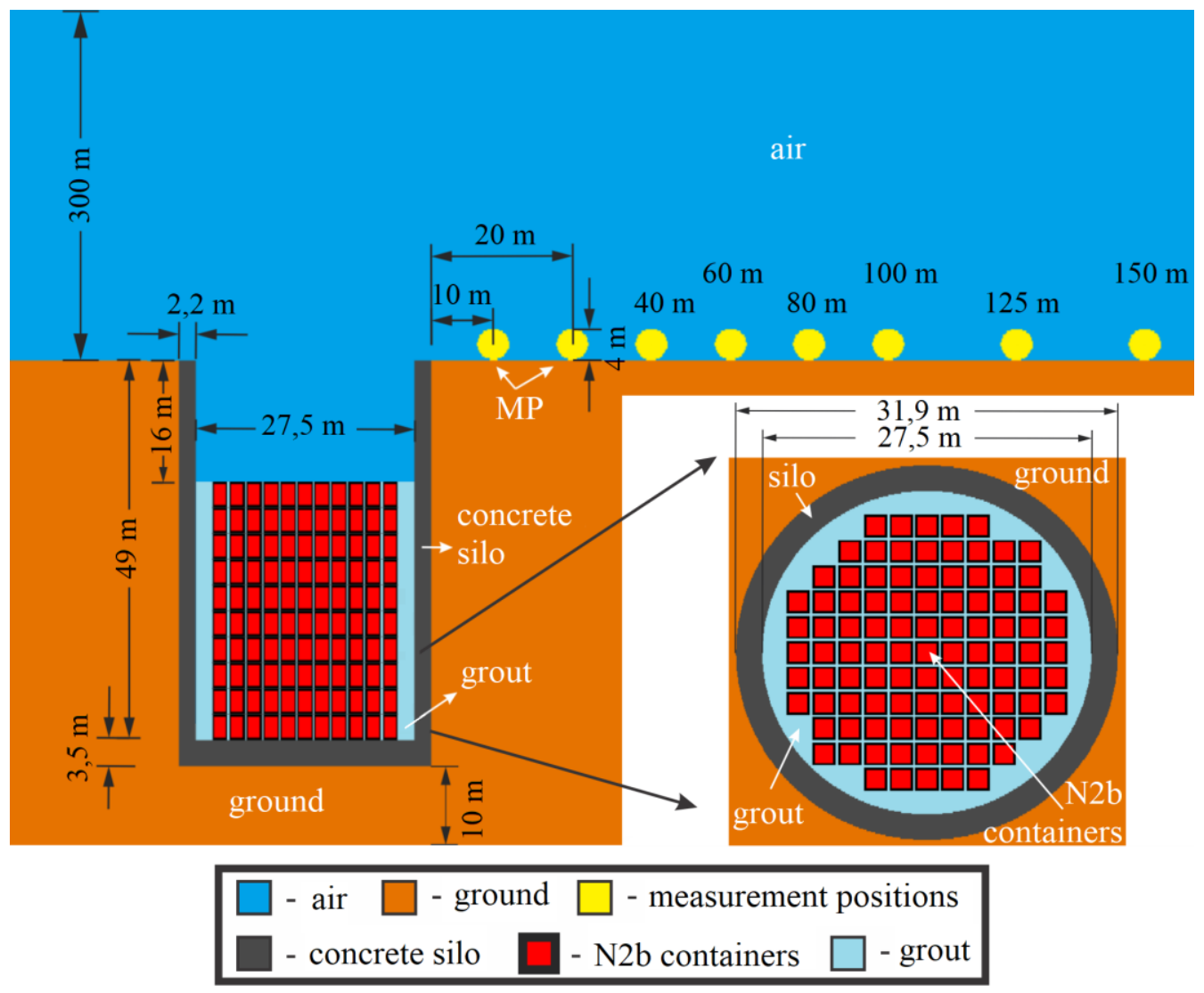

Figure. 1. Schematic view of the computational model of the LILW repository, side view (left) and top view (right). Due to clarity, four measurement positions are not visible (MP: $30 \mathrm{~m}, 50 \mathrm{~m}, 70 \mathrm{~m}$ and $90 \mathrm{~m}$ ). (drawing not to scale)

The radioactive waste was fully enclosed in standardized $\mathrm{N} 2 \mathrm{~b}$ disposal containers [10] with dimensions of $1.95 \mathrm{~m} \times 1.95 \mathrm{~m} \times 3.3 \mathrm{~m}$ and with the concrete wall thickness of $20 \mathrm{~cm}$ on the side and $22 \mathrm{~cm}$ on the top/bottom. The containers were placed $0.2 \mathrm{~m}$ from each other, where the entire intermediate space up to the inner wall of the silo was filled with grout. Also, containers were placed directly on top of each other without any intermediate space or fillings. To simplify calculations and the model, the entire radioactive waste, together with the filling grout, was homogenized and placed inside a concrete container with a volume of $1.55 \mathrm{~m} \times 1.55 \mathrm{~m} \times 2.86 \mathrm{~m}$. Such simplification is appropriate since the content of other materials in LILW, i.e. ash, other metal alloys, cellulose, plastic, etc., is negligible (less than $3 \%$ ). The simplified model of the N2b container is shown in Fig. 2 where the concrete wall and the homogeneous mixture (LILW + grout), which was considered as the source of particles in simulations, are presented as grey and red respectively. At this point, it should be emphasized that abovementioned model simplification is a conservative approach and corresponds to the extreme case of the N2b disposal container where the homogeneous mixture will be distributed throughout all of the inner space of the container. In general, barrels, in which this homogenous mixture will be placed, are slightly smaller and therefore an additional shield (e.g. filling grout) can be placed to fill the mentioned gap $(1 \mathrm{~cm}-19 \mathrm{~cm})$. 


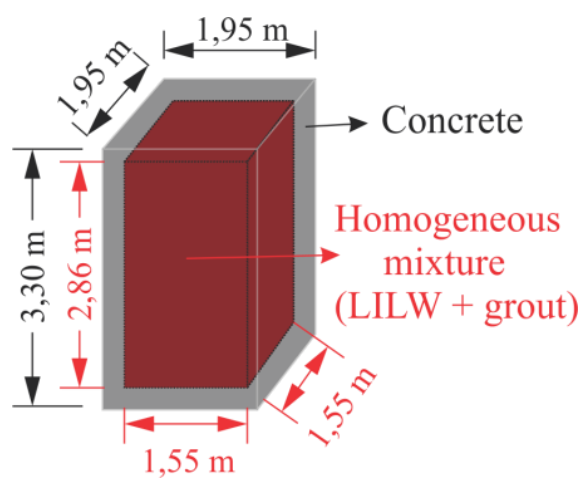

Figure. 2. The simplified model of the standardized N2b disposal container with the homogeneous mixture of LILW and grout (red) placed in the centre of the concrete construction (grey).

\subsection{Gamma-ray source definition}

In the simulation, only $\gamma$-rays originating in the waste were considered and transported accordingly as the range of $\alpha$ and $\beta$-particles is negligible. The source intensity was determined based on the detailed isotopic composition of the material which was obtained from the technical report [1].

In this analysis, two different waste sources were examined. In the first case, only isotopic composition due to decommissioning of the JSI TRIGA Mark II research reactor and waste stored in the Central Interim Storage (CIS) were considered. The total amount of these waste approximately corresponds to the amount of waste, which will be firstly stored in one layer $\left(1.342 \cdot 10^{13} B q\right.$ per 117 containers $)$. Energy and probability of produced $\gamma$-rays had to be considered due to multiple-stage radionuclides decay. The decay scheme data of individual isotope, i.e. the energy and probability of released $\gamma$-rays, were obtained from ENDF/B-VII.0 nuclear data library.

For a given case, the effective source intensity per layer of containers (99 containers) was $1.81 \cdot 10^{13} \frac{\gamma}{\mathrm{s}}$, whereby radionuclides with at least $0.1 \%$ contribution to activity were considered. The corresponding source spectrum, which presents the relative source intensity in dependence on the energy of the emitted $\gamma$ rays, is shown in Fig. 3 (left). The dominant proportion, $83 \%$, is due to the Cobalt ${ }^{60} \mathrm{Co}$ isotope with two characteristic $\gamma$ rays with energies of $1.17 \mathrm{MeV}$ and $1.33 \mathrm{MeV}$. It turned out that the mentioned cut-off in the activity had a negligible effect on the total $\gamma$-dose rates as the radionuclides, which were not included, also emit low energy $\gamma$-rays.

For the second case also waste source from Krško NPP (operational and decommissioning waste) was considered. These represent the major contribution both by activity and volume. Based on the technical report [1] radionuclides with the highest activity are ${ }^{55} \mathrm{Fe}\left(1.1 \cdot 10^{17} \mathrm{~Bq}\right),{ }^{60} \mathrm{Co}\left(9 \cdot 10^{16} \mathrm{~Bq}\right)$ and ${ }^{63} \mathrm{Ni}$ $\left(3 \cdot 10^{16} \mathrm{~Bq}\right)$, whereby all other radionuclides had at least one order of magnitude lower activity than ${ }^{60} \mathrm{Co}$. The results have shown, Fig. 3 (right), that the spectrum could be simplified by considering only ${ }^{60} \mathrm{Co}$ with two high energy characteristic $\gamma$-rays, whereby the contribution of all other radionuclides ${ }^{55} \mathrm{Fe}\left(E_{\gamma} \sim 5.9\right.$ $\mathrm{keV}$ ) and ${ }^{63} \mathrm{Ni}$ (beta-decay, demanding transport for electrons) are negligible. This simplification is based on analyzing the influence of the $\gamma$-rays energies on the overall $\gamma$-dose rates for five additional monoenergetic sources, which were compared to the original spectrum (waste from JSI + CIS), in dependence on the distance from the repository (12 measurement positions). To properly considered high energy $\gamma$-rays with low probability, the mentioned analysis was performed with monoenergetic energies up 
to $5 \mathrm{MeV}$. It is observed that the energy of a transported $\gamma$-rays plays a crucial role, whereby low energy $\gamma$ rays $(0.1 \mathrm{MeV})$ had more than six orders of magnitude lower contribution compared to the $1 \mathrm{MeV}$ monoenergetic source.
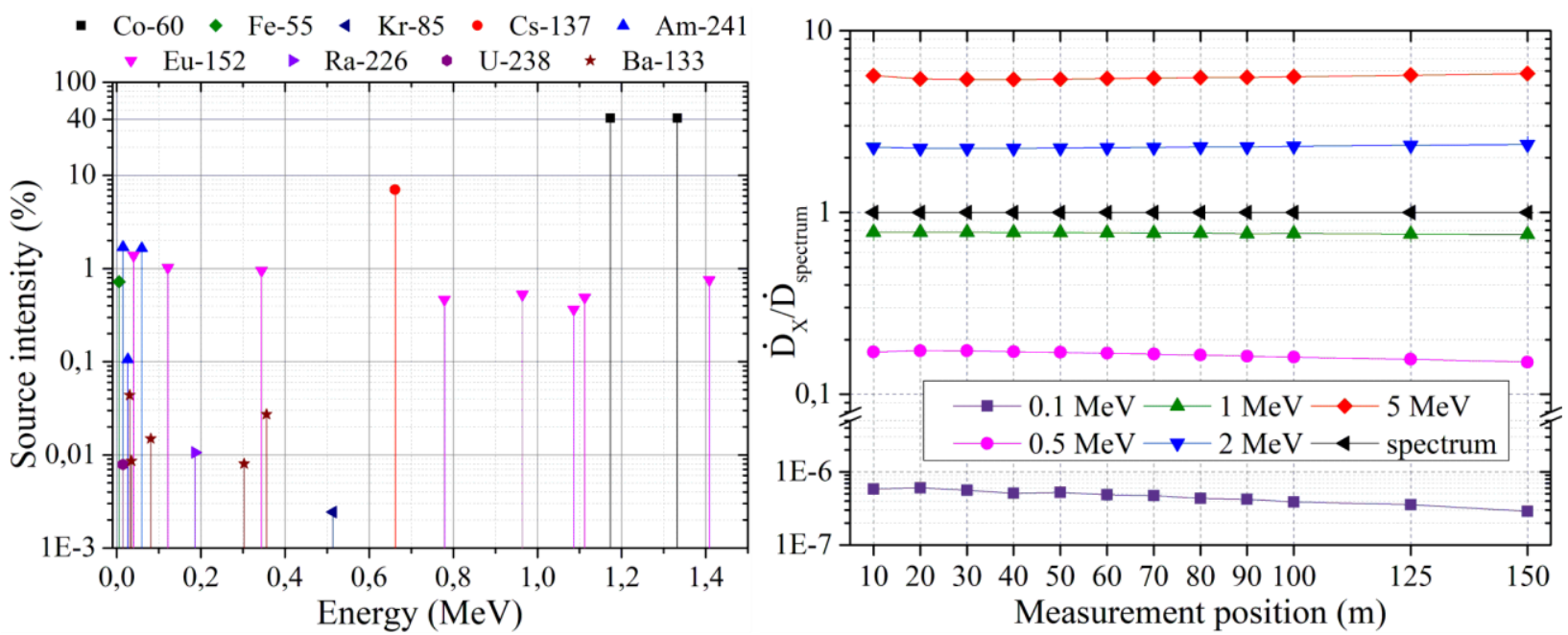

Figure. 3. Source line spectrum (waste from JSI + CIS), presenting relative source intensity in dependence on the energy of the emitted $\gamma$-rays (left). The influence of the $\gamma$-rays energies on the overall $\gamma$-dose rates for 12 measurement positions, whereby the results are normalized to the values obtained by the source spectrum

(right).

Based on these findings, the source spectrums for waste originating from JSI and CIS (JSI/CIS) and waste due to Krško NPP, can be simplified. Additionally, when we compare the JSI/CIS and Krško NPP waste, the only difference is in the overall number of disposal containers (117 and 1728) and the total activity of ${ }^{60} \mathrm{Co}\left(8.84 \cdot 10^{12} \mathrm{~Bq}\right.$ and $\left.9 \cdot 10^{16} \mathrm{~Bq}\right)$. Based on that the Krško NPP waste has effectively 698.3 times higher activity per the same amount of volume as waste from JSI/CIS. Thus, all further analyses were performed with the source spectra using JSI/CIS waste and using efficiency factor (698.3) to examine cases with higher impact on the annual $\gamma$-dose rate.

\section{RESULTS}

\subsection{ADVANTG - speed up}

The knowledge gained from the sensitivity study of a different ADVANTG input parameters, i.e. quadrature set, scattering order, geometrical mesh, multi-group data libraries etc., and effects such as air density, height of air above the radiation source etc., which was performed as part of the skyshine benchmark experiment paper [10], was used for further analyses and optimization. Based on those findings, the optimal ADVANTG parameters were chosen (parameters $S_{N}$ and $P_{n}$ were kept at the default values of 4 and 3 respectively), by which the hybrid simulation (ADVANTG + MCNP) achieved much higher relative efficiency (relative figure-of-merit) and accelerated analog MCNP simulation by factor from 1000 to 9500 depending on the distance from the repository (12 MPs: $10 \mathrm{~m}$ to $150 \mathrm{~m}$ from the outer edge of the repository). Since the number of the simulated particles reaching the farthest tallies decreases in the analog simulation the variance reduction becomes more effective with an increase in the distance from the repository. With the help of the optimized hybrid simulation all further analyses were achieved in a reasonable time (approx. 5 to 6 hours using 2 Intel Xeon E5-2680 v2 processors with $2 \times 10(2.8 \mathrm{GHz})$ cores resulting in 40 processor threads, and 128 GB of DDR3 memory). 


\subsection{Repository filling fraction}

To examine $\gamma$-dose rates during different stages of the repository filling, 4 different repository configurations were examined in more detailed: lvl-1, lvl-4, lvl-7 and lvl-10. Each configuration represents the fraction of the overall repository filling capacity, i.e. repository filling fraction: $10 \%, 40 \%, 70 \%$ and $100 \%$ respectively. In all cases an open repository was examined, shown in Fig. 4 (left), where only the highest layer was filled with containers (a simplified one-layer model), as the contribution of all lower layers was negligible (less than $10^{-4}$ ). The results of the $\gamma$-dose rate values for different repository filling are shown in Fig. 4 (right), where the dose rates are normalized to the values obtained by the reference case lvl-1. By increasing the position of the highest layer, or by increasing the level of the repository filling, the $\gamma$-dose rate values increased. For the individual case, the normalized values are approximately constant and distance-independent, where the discrepancies are less than $2 \%$. For the fully loaded open repository lvl-10, when the repository is filled to the tenth, last layer, the $\gamma$-dose values increased for a factor of 4 compared to the reference case lvl-1.
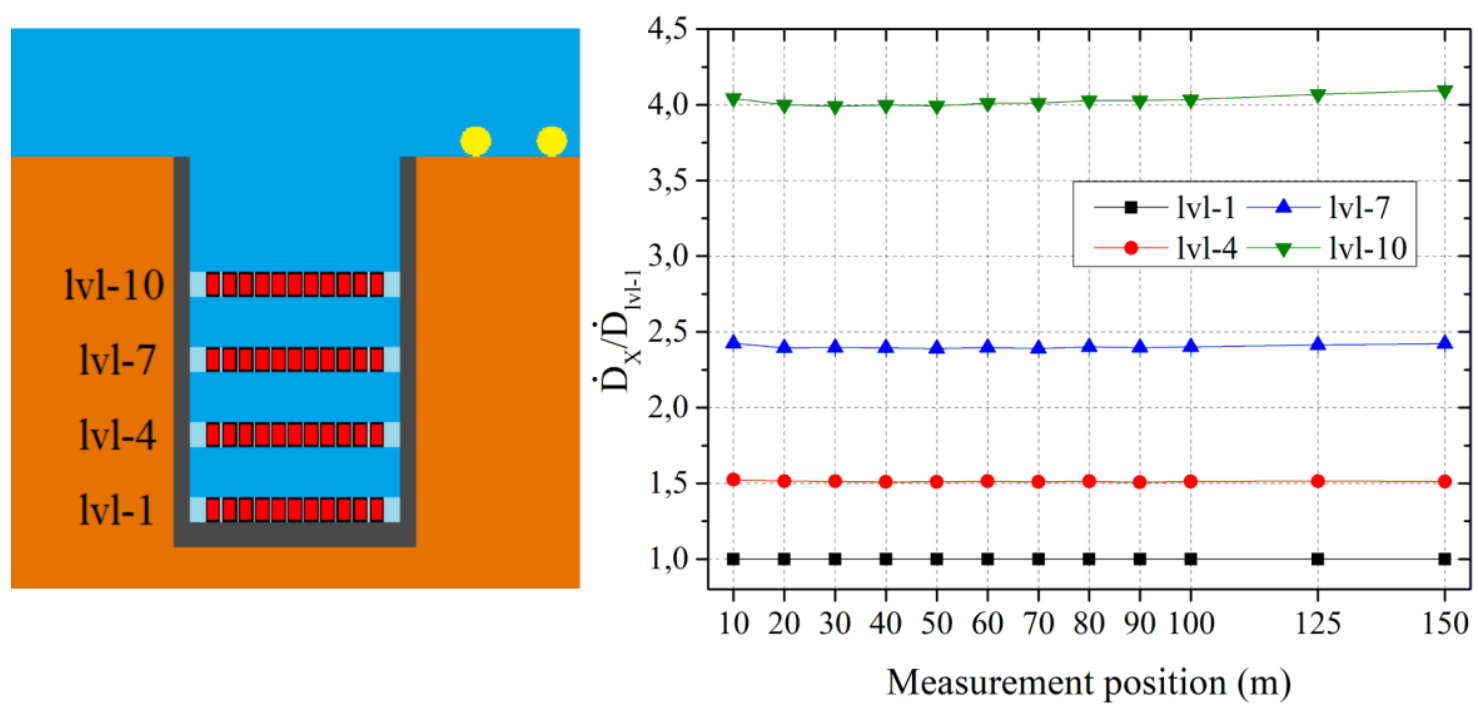

Figure. 4. The simplified one-layer model for 4 different repository configurations: lvl-1, Ivl-4, Ivl-7and lvl-10 (left) and their relative influence of the $\gamma$-dose rate values in dependence on 12 measurement positions (right).

\subsection{Annual $\gamma$-dose rate values}

Accurate calculation of the $\gamma$-dose rate around the LILW repository is crucial for safety analysis of closed as well as an open repository.

Calculated annual $\gamma$-dose rate values in dependence on 12 MPs are shown in Fig. 5 for 4 different repository configurations lvl-1, lvl-4, lvl-7 and lvl-10. Results for almost all configurations and MPs were higher than the reference limit value, which is $20 \mathrm{mSv} / \mathrm{year}$. The results show that the maximum calculated annual $\gamma$ dose rate value $723.8 \mathrm{mSv} /$ year was obtained for the case with fully loaded repository lvl-10 and nearest MP $(10 \mathrm{~m})$. With an increase of the distance from the repository, the calculated annual $\gamma$ dose rates were decreased, whereby the results at the last MP $150 \mathrm{~m}$ were 31 times lower compared to the results at the nearest MP $10 \mathrm{~m}$. 


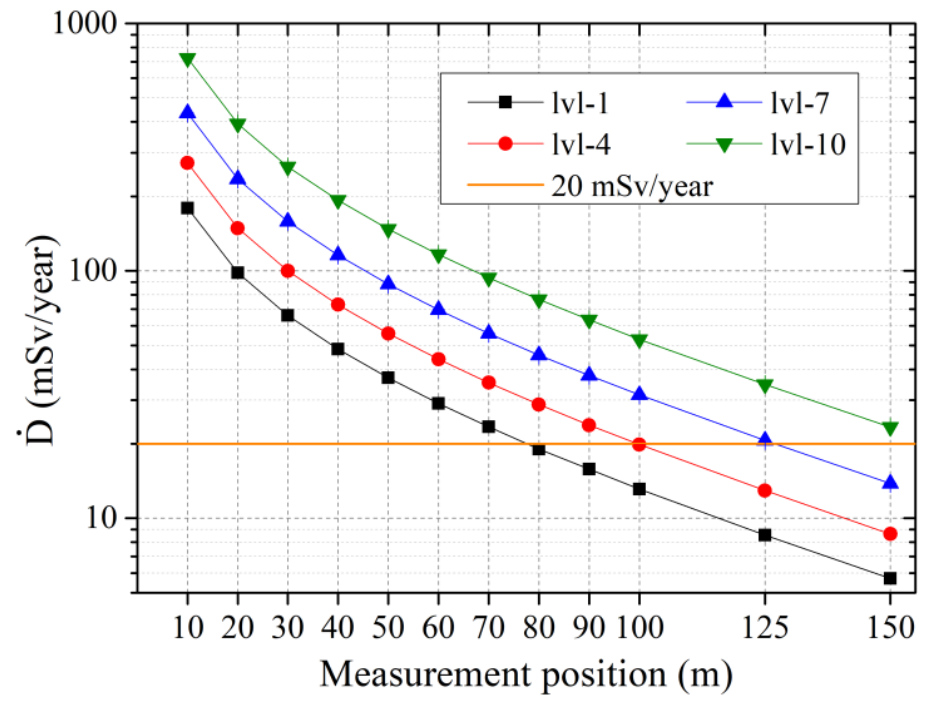

Figure. 5. The annual $\gamma$-dose rate values in dependence on 12 MPs for 4 different repository configurations (lvl-1, lvl-4, lvl-7 and lvl-10). For the comparison, limiting dose value for a professional worker is shown (20 mSv/year - full line).

Despite conservative assumptions in the repository model calculations indicate exceeding of annual dose limits for professionals. The main assumption used in the model was the homogeneous mixture of waste and the filling grout (shown as red in Fig. 2) inside the concrete N2b disposal container. This is a conservative simplification as the mixture of the filling grout and radioactive waste will first be inserted into the barrels of various dimensions and only then the barrels are inserted into the disposal container. In addition, the entire intermediate space between the barrels and inside wall of the $\mathrm{N} 2 \mathrm{~b}$ disposal container will be filled with a filling grout.

Thus, an additional analysis was performed, which involved a more detailed model of the N2b disposal container. Due to various sizes of the mentioned barrels, in which the homogenous mixture was placed, an additional fraction of the filling grout at the top of the homogeneous mixture was considered $(11 \mathrm{~cm}-44 \mathrm{~cm})$. The results with an additional $33 \mathrm{~cm}$ thick layer of filling grout show, that calculated annual $\gamma$-dose rate values for all configurations and MPs dropped below the prescribed reference limitation (20 mSv/year inside of the LILW site and $1 \mathrm{mSv} /$ year outside of the LILW site for the general population).

However, the required $33 \mathrm{~cm}$ thickness of additional filling grout cannot be applied to all cases due to overall container size limitations. Based on that, an analysis will be performed in the future to determine the maximum permissible height of the homogeneous mixture (waste + grout) for each type of the barrel. With this restriction, the total number of all required $\mathrm{N} 2 \mathrm{~b}$ disposal containers will increase. To maintain the overall number of containers, an additional thick layer of the shield should be added at the top of the barrels. However, the mentioned analysis has not been performed as part of this research.

\section{CONCLUSIONS}

In order to estimate $\gamma$-dose rates and other physical parameters used in safety assessment for the future Slovenian LILW repository, which is a unique case of a silo-type radiation facility, one has to use reliable and time-efficient codes. With ADVANTG and its variance reduction technique, detailed calculations of $\gamma$ dose rates around the repository were achievable in reasonable time, compared to all previous calculations which used a different kind of approximation, e.g. simplified deterministic methods, rough geometric 
models etc., which did not properly consider the skyshine effect, i.e. effect of backscattering in the air. ADVANTG has proven to be a powerful, easy to use tool for acceleration of Monte Carlo simulations in terms of the required CPU time and with relatively low user-time needed to produce effective variance reduction parameters. With the optimal ADVANTG calculation, a speed-up of analog simulation up to 9500 was achieved, which enabled a wide range of complex analyses in a reasonable time, which were not able with the standard analog methods.

According to our analysis, the source spectra could be simplified by considering only ${ }^{60} \mathrm{Co}$. Based on that, the Krško NPP waste has effectively 698.3 times higher activity per the same amount of volume as waste from JSI/CIS. Considering a case when the open repository was filled to the last tenth layer (lvl-10), the $\gamma$ dose values increased for a factor of 4 compared to the reference case, which included only first/lowest layer of containers (lvl-1). In addition, for the utmost case, fully loaded (lvl-10) and open repository at the nearest measurement position $(10 \mathrm{~m})$, the annual $\gamma$-dose rate was $723.8 \mathrm{mSv} /$ year $(82.6 \mu \mathrm{Sv} / \mathrm{h})$, which is greatly above the prescribed limit value $(20 \mathrm{mSv} / \mathrm{year})$. By considering a more detailed model of the concrete $\mathrm{N} 2 \mathrm{~b}$ disposal container, which includes an additional $33 \mathrm{~cm}$ thick layer of the filling grout on top of the homogeneous mixture (waste + grout), the calculated dose rate values drop below the limit values. It turned out, that the modelling practices play an important role. Furthermore, an additional analysis is proposed in the future where the model for the individual barrel type will be examined in more detail.

\section{ACKNOWLEDGMENTS}

The work was supported by the Slovenian Ministry of Education, Science and Sport (P2-0073 Reactor Physics).

\section{REFERENCES}

1. Slovenian Agency for Radioactive Waste (ARAO), "Projektne osnove za odlagališče NSRAO Vrbina, Krško - faza presoje vplivov na okolje,” Technical report, NSRAO2-POR-013-02, 2018.

2. "Krško NPP" https://www.nek.si/en

3. "TRIGA" www.rcp.ijs.si/ric/index-a.htm

4. X-5 Monte Carlo Team. (2004). MCNP- A General Monte Carlo N. Particle Transport Code, Version 5, LA-UR-03-1987. Los Alamos National Laboratory.

5. S. W. Mosher, S. R. Johnson, A. M. Bevill, A. M. Ibrahim, C. R. Daily, T. M. Evans, J. C. Wagner, J. O. Johnson, and R. E. Grove, ADVANTG-An Automated Variance Reduction Parameter Generator, ORNL/TM-2013/416 Rev. 1, Oak Ridge National Laboratory, Oak Ridge, TN (2015).

6. J.C. Wagner, A. Haghighat, Automated Variance Reduction of Monte Carlo Shielding Calculations Using the Discrete Ordinates Adjoint Function, Nuclear Science and Engineering, 128, 186 (1998).

7. E. A. Belogorlov, G. I. Britvich, V. B. Getmanov, et al. Neutron fields investigation in three sectional concrete labyrinth from Cf-252 source. Submitted to: At. Energ., 1984.

8. D. Kotnik, A. Čufar, B. Kos, L. Snoj "Validation and Evaluation of the ADVANTG Hybrid Code on the ICSBEP Labyrinth Benchmark Experiment", Annals of Nuclear Energy, 2018.

9. O. F. Dikareva, I. A. Kartashev, M. E. Netecha in V. P. Zharkov. Baikal-1 skyshine experiment. International Handbook of Evaluated Criticality Safety Benchmark Experiments, 2016. ALARMREACAIR-SKY-1.

10. D. Kotnik, B. Kos, A. Čufar, et al. Validation and evaluation of the ADVANTG code on the ICSBEP skyshine benchmark experiment. Annals of Nuclear Energy, 125: 249 - 260, 2019.

11. M. B. Chadwick, M. Herman, P. Oblozinsky, et al., "ENDF/B-VII.1 nuclear data for science and technology: Cross sections, covariances, fission product yields and decay data", Nuclear Data Sheets, 112(12):2887-2996 (2011). 